\title{
NOTES
}

\section{A NOTE ON AN EXTENSION OF A CLASS OF SOLUTIONS TO DYNAMIC PROGRAMMING PROBLEMS ARISING IN ECONOMIC GROWTH}

\author{
JÜRGEN ANTONY
}

CPB Netherlands Bureau for Economic Policy Analysis

\author{
Alfred MAuBner \\ University of Augsburg
}

This note extends the findings of Benhabib and Rusticchini [Journal of Economic Dynamics and Control 18, 807-813 (1994)], who provide a class of dynamic stochastic general equilibrium (DSGE) models whose solution is characterized by a constant savings rate. We show that this class of models may be interpreted as a standard-representative agent DSGE model with costly adjustment of capital.

Keywords: Capital and Labor Substitution, Dynamic Programming, Growth, Numerical Solutions of DSGE Models

\section{INTRODUCTION}

Dynamic stochastic general equilibrium (DSGE) models have become the standard tool for analyzing many questions in business cycle research, finance, growth, and monetary economics. Except for a few cases, the solutions of these models must be approximated by numerical methods. In these circumstances it is often very helpful to start from a model that is known to have an analytical solution and to approach the model of interest by way of homotopy methods [see Heer and Maußner (2009, Chap. 5 and 6)]. From this perspective, extensions of the class of models with analytic solutions are very valuable.

Benhabib and Rusticchini (1994), henceforth BR, extend the class of DSGE models that are known to have a solution in terms of a constant savings rate. They employ a model with two vintages of capital. In this note we first show that their specification is easily extended to the case of an infinite number of vintages 
and second reinterpret this specification in terms of a model with frictions in the adjustment of capital.

In the next section we present the model of BR as well as our extension and reinterpretation of it. Section 3 concludes, and the Appendix covers a few technical details.

\section{THE MODEL}

BR consider a representative agent with additively time-separable preferences who discounts future utility at a rate $\delta \in(0,1)$ and whose instantaneous utility function $u$ is given by

$$
u\left(c_{t}, 1-L_{t}\right)=\frac{A\left(c_{t}^{1-\epsilon}-1\right)}{1-\epsilon}+w\left(1-L_{t}\right),
$$

where $c$ denotes consumption and $L$ hours worked. $A>0$ and $\epsilon \geq 0$ are given parameters. $w$ is a concave, increasing function. The agent employs labor and two vintages of capital, $k_{1}$ and $k_{2}$, respectively, to produce output according to

$$
y_{t}=z_{t}\left[a_{1} k_{1 t}^{1-\epsilon}+a_{2} k_{2 t}^{1-\epsilon}+\left(1-a_{1}-a_{2}\right) L_{t}^{1-\epsilon}\right]^{\frac{1}{1-\epsilon}} .
$$

$z$ is an i.i.d. productivity shock with support $[\underline{z}, \bar{z}] .{ }^{1}$ The agent's resource constraint is

$$
k_{1 t+1}=y_{t}-c_{t} .
$$

In addition, capital depreciates at a rate $\mu \in[0,1]$, so that

$$
k_{2 t+1}=\mu k_{1 t} \text {. }
$$

BR prove that $c_{t}=\lambda y_{t}$ is the agent's policy function for consumption, where

$$
(1-\lambda)=\left[a_{1} \delta \mathbf{E}_{t}\left(z_{t+1}^{1-\epsilon}\right)+a_{2} \delta^{2} \mu^{1-\epsilon} \mathbf{E}_{t}\left(z_{t+2}^{1-\epsilon}\right)\right]^{\frac{1}{\epsilon}}
$$

The crucial assumption that allows this solution is that the agent's preference parameter $\epsilon$ (his or her coefficient of relative risk aversion) equals the reciprocal of the elasticity of substitution of the production function.

As BR note, the extension to more than two vintages is straightforward. In the case of an infinite number of vintages $k_{j}$ being related to each other via

$$
k_{j+1 t+1}=\mu k_{j t},
$$

the production function (2) may be written as

$$
y_{t}=z_{t}\left[\sum_{j=1}^{\infty} a_{j} k_{j t}^{1-\epsilon}+\left(1-\sum_{j=1}^{\infty} a_{j}\right) L_{t}^{1-\epsilon}\right]^{1 /(1-\epsilon)}, \quad \sum_{j=1}^{\infty} a_{j}<1 .
$$


The general solution for the savings rate $1-\lambda$ at time $t$ is then given (see the Appendix) by

$$
1-\lambda=\left[\sum_{j=1}^{\infty} a_{j} \delta^{j} \mu^{(j-1)(1-\epsilon)} \mathbf{E}_{t}\left(z_{t+j}^{1-\epsilon}\right)\right]^{\frac{1}{\epsilon}} .
$$

An alternative interpretation of this framework is to use a traditional constant elasticity of substitution production function with labor $L_{t}$ and capital $K_{t}$ as inputs,

$$
y_{t}=z_{t}\left[\alpha L_{t}^{1-\epsilon}+(1-\alpha) K_{t}^{1-\epsilon}\right]^{\frac{1}{1-\epsilon}},
$$

and to assume adjustment costs of capital that give raise to the transition function

$$
K_{t}=\left[\beta K_{t-1}^{1-\epsilon}+(1-\beta) k_{t-1}^{1-\epsilon}\right]^{\frac{1}{1-\epsilon}},
$$

where $k_{t}$ denotes investment from foregone consumption in period $t$. Inserting (10) repeatedly into (9) yields

$$
y_{t}=\left[\alpha L_{t}^{1-\epsilon}+(1-\alpha) \beta \sum_{j=1}^{\infty}(1-\beta)^{j-1} k_{t-j}^{1-\epsilon}\right]^{\frac{1}{1-\epsilon}} .
$$

This production technique equals (7) if $a_{j} \mu^{(j-1)(1-\epsilon)}=(1-\alpha) \beta(1-\beta)^{j-1}$. Thus, the savings rate at time $t=0$ is given by

$$
1-\lambda=\left[\sum_{j=1}^{\infty}(1-\alpha) \beta(1-\beta)^{j-1} \delta^{j} \mathbf{E}_{t}\left(z_{t+j}^{1-\epsilon}\right)\right]^{\frac{1}{\epsilon}} .
$$

\section{CONCLUSION}

DSGE models featuring an analytical solution are helpful for applied researchers because they can use this solution as a starting point for the computation of the solutions of more complicated models. In this note, we have shown that the class of DSGE models provided by BR can be interpreted as a more traditional DSGE model with adjustment costs of capital. This interpretation can also be seen as a generalization of the well-known closed-form solution to the Ramsey problem [see, e.g., McCallum (1989)] with log utility, Cobb-Douglas production, and a capital accumulation equation given by $K_{t}=K_{t-1}^{\beta} k_{t-1}^{1-\beta}$ arising from (10) if $\epsilon$ equals unity.

\section{NOTES}

1. BR, p. 809, assume that $z$ follows a first-order autoregressive process. However, as they note on p. 811 , the savings rate $\lambda$ will be constant only if $z$ is i.i.d. 
2. Note that this definition implies the parameter restriction $\bar{z}^{1-\epsilon} \sum_{j=1}^{\infty} a_{j}<1$, because

$$
k=\left[\frac{1-\sum_{j=1}^{\infty} a_{j}}{1-\bar{z}^{1-\epsilon} \sum_{j=1}^{\infty} a_{j}}\right]^{\frac{1}{1-\epsilon}} .
$$

Because $z$ implicitly defines the scale for output, this condition is not restrictive.

3. More explicitly, let $k_{1}^{1}=h\left(k_{1}, \ldots, k_{n}, z\right)$ denote the solution of the first-order condition (A.1). Then, the Bellman equation can be written as

$$
v\left(k_{1}, \ldots, k_{n}, z\right)=\frac{A\left[y-h\left(k_{1}, \ldots, k_{n}, z\right)^{1-\epsilon}\right]}{1-\epsilon}+\delta \mathbf{E} V\left(k_{1}^{1}, \mu k_{1}, k_{3}^{1}, \ldots, k_{n}^{1}, z^{1}\right) .
$$

Differentiating with respect to $k_{1}$ yields

$$
\begin{aligned}
& V_{1}\left(k_{1}, \ldots, k_{n}, z\right)=A c^{-\epsilon} y_{1}+\mu \delta \mathbf{E} V_{2}\left(k_{1}^{1}, \ldots, k_{n}^{1}, z^{1}\right)+h_{1}(\cdot)\left\{\left[\delta V_{1}\left(k_{1}^{1}, \ldots, k_{n}^{1}, z^{1}\right)\right]-A c^{-\epsilon}\right\} \\
& \quad=A c^{-\epsilon} y_{1}+\mu \delta \mathbf{E} V_{2}\left(k_{1}^{1}, \ldots, k_{n}^{1}, z^{1}\right),
\end{aligned}
$$

because the term in braces vanishes due to the first-order condition (A.1). In the same way, one can derive $V_{2}\left(k_{1}, \ldots, k_{n}, z\right)=A c^{-\epsilon} y_{2}+\mu \delta \mathbf{E} V_{3}\left(k_{1}^{1}, \ldots, k_{n}^{1}, z^{1}\right)$, etc.

\section{REFERENCES}

Benhabib, J. and A. Rustichini (1994) A note on a new class of solutions to dynamic programming problems arising in economic growth. Journal of Economic Dynamics and Control 18, 807-813.

Heer, B. and A. Maußner (2009) Dynamic General Equilibrium Models: Computational Methods and Applications, 2nd ed. Berlin: Springer.

McCallum, B.T. (1989) Real business cycle models. In R. Barro (ed.), Modern Business Cycle Theory. Cambridge, MA: Harvard University Press.

\section{APPENDIX}

First, we show that there is an upper bound on output such that consumption $c$ and (via the continuity of $u(c, 1-L)$ ) expected lifetime utility are finite. Let $^{2}$

$$
k=\bar{z}\left[\sum_{j=1}^{\infty} a_{j} k^{1-\epsilon}+1-\sum_{j=1}^{\infty} a_{j}\right]^{\frac{1}{1-\epsilon}}
$$

denote investment if $z_{t}=\bar{z}, k_{j t}=k, L_{t}=1, c_{t}=0$. If $k_{1 t+1}=k$ and $c_{t+1}=0$, then

$$
\begin{aligned}
& k_{1 t+2}=y_{t+1}=z_{t}\left[a_{1} k^{1-\epsilon}+a_{2}(\mu k)^{1-\epsilon}+\cdots+\left(1-\sum_{j=1}^{\infty} a_{j}\right) L_{t}^{1-\epsilon}\right]^{\frac{1}{1-\epsilon}} \\
& \leq k=\bar{z}\left[a_{1} k^{1-\epsilon}+a_{2} k^{1-\epsilon}+\cdots+\left(1-\sum_{j=1}^{\infty} a_{j}\right)\right]^{\frac{1}{1-\epsilon}} .
\end{aligned}
$$

Because $k_{1 t+2}$ cannot exceed $k, y_{t+2}$ cannot exceed $y=k$, so $k_{1 t+s} \leq k$ for all $s=1,2, \ldots$. Thus, output, investment, and, hence, consumption are bounded. 
Second, we derive the optimality conditions for an arbitrarily given number of vintages $n=1,2, \ldots$ We focus on the condition with respect to investment $k_{1 t+1}$, because the optimization with respect to labor input $L$ implies a static first-order condition that can be solved for $L$ given the sequence of vintages without knowledge of consumption $c$. Let $v\left(k_{1}, k_{2}, \ldots, k_{n}, z\right)$ denote the value function, i.e., the maximum expected lifetime utility of an optimal policy given the initial conditions $\left(k_{1}, k_{2}, \ldots, k_{n}, z\right)$. This function is recursively defined by the Bellman equation,

$$
v\left(k_{1}, k_{2}, \ldots, k_{n}, z\right)=\max _{k_{1}^{1}} \frac{A\left[\left(y-k_{1}^{1}\right)^{1-\epsilon}-1\right]}{1-\epsilon}+\delta \mathbf{E} V\left(k_{1}^{1}, k_{2}^{1}, \ldots, k_{n}^{1}, z^{1}\right),
$$

where $x^{i}, i=1,2, \ldots$, denotes a variable, which is $i$ periods ahead, and $\mathbf{E}$ is the conditional expectations operator. The first-order condition for the maximization problem on the righthand side of the Bellman equation is

$$
A c^{-\epsilon}=\delta \mathbf{E} V_{1}\left(k_{1}^{1}, \ldots, k_{n}^{1}, z^{1}\right),
$$

where $V_{i}$ denotes the derivative of $V$ with respect to its $i$ argument. The envelope theorem implies that $V_{1}$ is recursively defined $\mathrm{via}^{3}$

$$
V_{i}\left(k_{1}, \ldots, k_{n}, z\right)=A c^{-\epsilon} y_{i}+\mu \delta \mathbf{E} V_{i+1}\left(k_{1}^{1}, \ldots, k_{n}^{1}, z^{1}\right)
$$

with initial condition

$$
V_{n}\left(k_{1}, \ldots, k_{n}, z\right)=A c^{-\epsilon} y_{n},
$$

where $y_{i}$ denotes the derivative of the production function

$$
y=z\left[\sum_{j=1}^{n} a_{j} k_{j}^{1-\epsilon}+\left(1-\sum_{j=1}^{n} a_{j}\right) L^{1-\epsilon}\right]^{\frac{1}{1-\epsilon}}
$$

with respect to vintage $i=1,2, \ldots, n$, which is equal to

$$
y_{i}(\cdot)=z^{1-\epsilon}\left(\frac{k_{i}}{y}\right)^{-\epsilon}
$$

Using (A.2a) and (A.3), the first-order condition can be written as

$$
A c^{-\epsilon}=\mathbf{E}\left\{\sum_{i=1}^{n} a_{i} \delta^{i} \mu^{i-1}\left(c^{i}\right)^{-\epsilon}\left(z^{i}\right)^{1-\epsilon}\left(\frac{k_{i}^{i}}{y^{i}}\right)^{-\epsilon}\right\} .
$$

Assume $k_{1}^{1}=(1-\lambda) y$ so that $c=\lambda y$ and note that

$$
k_{i}^{i}=\mu^{i-1} k_{1}^{1}=\mu^{i-1}(1-\lambda) y .
$$

Thus, the first-order condition (A.4) reduces to

$$
1=(1-\lambda)^{-\epsilon} \mathbf{E}\left\{\sum_{i=1}^{n} a_{i} \delta^{i} \mu^{(i-1)(1-\epsilon)}\left(z^{i}\right)^{1-\epsilon}\right\} .
$$

Obviously, the savings rate $\lambda$ defined in equation (8) is the limit of (A.5) for $n \rightarrow \infty$ (and $z^{i}$ replaced with $z_{t+j}$.) 